\title{
Sensing bacterial infections by NAIP receptors in NLRC4 inflammasome activation
}

\author{
Yi-Nan Gong ${ }^{1}$, Feng Shao ${ }^{2}$ \\ ${ }^{1}$ National Institute of Biological Sciences, Beijing 102206, China \\ ${ }^{2}$ Howard Hughes Medical Institute, National Institute of Biological Sciences, Beijing 102206, China \\ $\square$ Correspondence: shaofeng@nibs.ac.cn \\ Received February 7, 2012 Accepted February 8, 2012
}

\begin{abstract}
The inflammasome is an emerging new pathway in innate immune defense against microbial infection or endogenous danger signals. The inflammasome stimulates activation of inflammatory caspases, mainly caspase-1. Caspase-1 activation is responsible for processing and secretion of IL-1 $\beta$ and IL-18 as well as for inducing macrophage pyroptotic death. Assembly of the large cytoplasmic inflammasome complex is thought to be mediated by members of NOD-like receptor (NLR) family. While functions of most of the NLR proteins remain to be defined, several NLR proteins including NLRC4 have been shown to assemble distinct inflammasome complexes. These inflammasome pathways, particularly the NLRC4 inflammasome, play a critical role in sensing and restricting diverse types of bacterial infections. Here we review recent advances in defining the exact bacterial ligands and the ligand-binding receptors involved in NLRC4 inflammasome activation. Implications of the discovery of the NAIP family of inflammasome receptors for bacterial flagellin and type III secretion apparatus on future inflammasome and bacterial infection studies are also discussed.
\end{abstract}

KEYWORDS inflammasome, NOD-like receptors, NLRC4, caspase-1, NAIP, type III secretion system, flagellin, Salmonella, Legionella, enteropathogenic E. coli, Burkholderia

\section{INTRODUCTION OF INFLAMMASOME IN INNATE IMMUNITY}

The innate immune system in mammals defends against microbial infection. To discriminate "microbial foreigners" from "self," the innate immune system has evolved an array of germline-encoded pattern recognition receptors (PRRs), which exhibits a broad specificity to a wide spectrum of pathogen-associated molecular patterns (PAMPs) (Takeuchi and Akira, 2010). Engagement of PAMPs by the cognate PRRs mounts an inflammatory response, exemplified by expression and secretion of a plethora of inflammatory cytokines, which functions to restrict microbial infection and promote subsequent adaptive immune response. The membrane-bound Toll-like receptors (TLRs) are established to directly recognize specific microbial products and thereby stimulate the proinflammatory NF- $\mathrm{KB}$ and interferon signaling, leading to transcriptional induction of many cytokines. The cytosolic NOD-like receptors (NLRs) (Chen et al., 2009) are another family of PRRs that are also thought to function to sense microbial products, serving as an intracellular innate immune surveillance mechanism. NLR proteins generally contain an $\mathrm{N}$-terminal caspase recruitment domain (CARD) or a pyrin domain, a central nucleotide-binding and oligomerization domain (NOD) and a C-terminal leucine-rich repeat (LRR) domain. This structural organization is similar to Apaf-1 in apoptosis as well as plant disease resistance $(R)$ gene product, the latter of which mediates innate immune defense in plants. In mammals, the NLR family contains 23 members in human and 34 members in mice (Ting et al., 2006, Davis et al., 2011).

NLR proteins can mediate the assembly of the so-called inflammasome complex mainly in macrophage or dendritic cells. The inflammasome acts as a signaling platform for inducing caspase-1 autoprocessing and activation, which further leads to maturation and secretion of IL-1 $\beta$ and IL-18 (Schroder and Tschopp, 2010). Caspase-1 activation often triggers a special type of macrophage inflammatory death termed pyroptosis (Fink and Cookson, 2006; Brodsky and 
Medzhitov, 2011). Both interleukin production and macrophage pyroptosis serve as effector mechanisms in limiting bacterial replication and defending against infection (Miao et al., 2010a). The CARD or PYD domains in NLR proteins signal downstream caspase-1 activation through direct CARD-CARD interaction with caspase-1 or indirectly through the inflammasome adaptor protein ASC. Similarly to Apaf-1 in apoptosome, NLR protein, upon sensing of the PAMP signal or certain endogenous danger signal, oligomerizes to form the large inflammasome complex, which requires nucleotide binding to its NOD domain (Duncan et al., 2007; Faustin et al., 2007). Four NLR proteins including NLRP3 (Anand et al., 2011), NLRP6 (Chen et al., 2011; Elinav et al., 2011), NLRC4 and mouse Nalp1b (Boyden and Dietrich, 2006), together with a non-NLR protein AIM2 (Hornung and Latz, 2010), have been demonstrated to function in the inflammasome pathway and play important but distinct roles in detecting and counteracting bacterial infection.

NLRC4 bears an N-terminal CARD domain that activates caspase-1 and downstream inflammasome signaling in both ASC-dependent and -independent manners (Broz et al., 2010b). Earlier genetic studies have established that NLRC is responsible for sensing cytosolic presence of bacterial flagellin in macrophage cells (Franchi et al., 2006; Miao et al., 2006). Recent studies on the function and mechanism of NLRC4 inflammasome activation have identified components of bacterial type III secretion apparatus as additional bacterial ligands for NLRC4 inflammasome and also the NAIP-family NLR proteins as the corresponding inflammasome receptors, which are the focus of the present review.

\section{BACTERIAL TYPE III SECRETION SYSTEM AND BACTERIAL INFECTION}

Bacterial pathogens and their eukaryotic hosts have been engaged in a constant battle during evolution. Bacterial pathogens usually harbor specialized secretion systems to inject virulence effector proteins into host cells to manipulate or disrupt various host processes, particularly the innate immune defense pathway. Many Gram-negative bacterial pathogens, such as Yersinia spp. (plague and gastrointestinal disorders), Salmonella spp. (typhoid or non-typhoid fever), Shigella spp. (bacillary dysentery), food-poisoning pathogenic Escherichia coli, Burkholderia spp. (glanders in human and animals), Pseudomonas spp. (that can infect humans, animals and plants), and the rare human pathogen Chromobacterium violaceum (that potentially can cause liver abscesses and sepsis), all harbor a so-called type III secretion system (Galán and Wolf-Watz, 2006; Cornelis, 2010). The type III secretion system is an essential virulence mechanism for these pathogens as it usually delivers multiple effectors with extremely potent and harmful activities into host cells (Cui and Shao, 2011). The type III secretion system, composed of more than 20 different protein subunits, is a sophis- ticated translocation apparatus characterized by a multi-ring base and an inner rod (Marlovits et al., 2006), located on the bacterial membranes and periplasm, as well as a protruding needle that forms a conduit for effector secretion (Deane et al., 2006). Many of the type III secretion apparatus subunits share sequence and structural similarity to components of bacterial flagellar export system that translocates monomeric flagellin out of the bacteria for flagellar assembly. Therefore, it is generally believed that two systems are evolutionarily related (Erhardt et al., 2010). Although type III secretion-containing bacterial pathogens, except for Shigella flexneri that is nonflagellar but does harbor a flagellin-encoding gene, usually come with the flagellar appendage for motility, there are also flagellar bacteria that do not harbor the type III secretion system. For example, Legionella pneumophila, the causative agent of Legionnaires' pneumonia and a model intracellular pathogen, features a type IV secretion system that is required for $L$. pneumophila replication within host alveolar macrophage cells (Isberg et al., 2009; Ge and Shao, 2011).

\section{FLAGELLIN RECOGNITION BY NAIP5(6) RECEPTORS}

Earlier studies showed that several Salmonella spp. are cytotoxic to macrophage or macrophage-like cells (Chen et al., 1996). Salmonella infection triggers caspase-1 activation, and blocking caspase-1 activation by a caspase-1-specific inhibitor or genetic ablation inhibits Salmonella infection-induced macrophage death (Hersh et al., 1999), suggesting inflammasome activation upon macrophage sensing of Salmonella infection. Salmonella-induced inflammasome activation entails the NLR protein NLRC4 as bone marrow macrophages derived from NIrc4 knockout mice show no caspase-1 activation and can survive Salmonella infection (Mariathasan et al., 2004). On the bacterial side, the gene encoding the monomeric flagellin, the building blocks of bacterial flagella, is responsible for NLRC4-dependent caspase-1 activation (Franchi et al., 2006; Miao et al., 2006; Broz et al., 2010a). Mutant S. typhimurium strain lacking flagellin is deficient in activation of caspase-1 and IL-1 $\beta$ secretion; cytoplasmic delivery of purified flagellin into macrophages by cationic liposome or pore-forming toxins can trigger NLRC4 inflammasome activation (Franchi et al., 2006; Miao et al., 2006; Molofsky et al., 2006). Consistent with the wide presence of the flagella among bacterial species, NLRC4 inflammasome-mediated innate immune detection has been observed with many other types of bacterial pathogens including Pseudomonas aeruginosa (Franchi et al., 2007; Sutterwala et al., 2007; Galle et al., 2008; Miao et al., 2008), L. pneumophila (Amer et al., 2006; Molofsky et al., 2006; Ren et al., 2006; Case et al., 2009), and the Gram-positive Listeria monocytogenes (Warren et al., 2008; Tsuchiya et al., 2010; Wu et al., 2010). However, a defined biochemical assay us- 
ing the amino-terminal domain of anthrax lethal factor-mediated intracellular delivery revealed that recombinant flagellin derived from several bacterial species, including enteropathogenic E. coli (EPEC), enterohemorrhagic E. coli (EHEC), S. flexneri, C. violaceum and Burkholderia thailandensis, is biochemically inactive in stimulating NLRC4 inflammasome activation (Zhao et al., 2011). Consistently, genetic ablation of flagellin-encoding genes from EPEC, $C$. violaceum and $B$. thailandensis has little effects on infection-induced caspase-1 activation. Thus, although flagellin detection appears to be a conserved mechanism for the host to recognize invading pathogenic bacteria, certain bacterial species have escaped from this NLRC inflammasome-mediated innate immune detection likely due to modification of their flagellin sequence.

A flagellar mutant of $L$. pneumophila that expresses flagellin but does not assemble the flagellum still induces flagellin-dependent macrophage cell death (Ren et al., 2006), suggesting that inflammasome sensing of flagellin does not require an intact flagellum structure. It has been shown that the C-terminal D0 region of flagellin is responsible for activating the inflammasome pathway (Lightfield et al., 2008). In fact, the C-terminal 35 amino acids of $L$. pneumophila flagellin are required and sufficient for stimulating NLRC4 inflammasome activation. Mutation of three leucine residues that disrupts a hydrophobic patch within the 35 amino acid region also abolishes inflammasome-mediated detection of flagellin from L. pneumophila (Lightfield et al., 2008; Zhao et al., 2011) and $P$. aeruginosa (Franchi et al., 2007). The inflammasome recognition region in flagellin is distinct from that by the plasma membrane-bound TLR family member TLR5 that is established to be the flagellin receptor for activating the NF- $\kappa B$ signaling. TLR5 appears to recognize the D1 region of flagellin and an 1411A mutation in L. pneumophila flagellin that disrupts TLR5 binding is fully functional in activating NLRC4 inflammasome (Lightfield et al., 2008). Macrophages derived from TLR5 knockout mice are still responsive in flagellin stimulation of the inflammasome pathway (Franchi et al., 2006; Miao et al., 2006). This further suggests that TLR5 is not the flagellin receptor for inflammasome activation.

What is the cytosolic receptor for flagellin in inflammasome activation? NLRC4 was the most attractive candidate when the genetic relation between bacterial flagellin and NLRC4 was first established. However, direct binding of flagellin to NLRC4 has never been recorded in the literature. Two recent studies propose that NAIP5 (probably also NAIP6), a member of the BIR domain-containing NLR protein family, serves as the innate immune receptor for flagellin in inflammasome activation in mice (Kofoed and Vance, 2011; Zhao et al., 2011). NAIP5 was originally identified as the genetic determinant for controlling $L$. pneumophila replication in murine macrophages (Diez et al., 2003; Wright et al., 2003; Derré and Isberg, 2004; Fortier et al., 2007). A natural Naip5 mutant allele renders macrophages from the $\mathrm{A} / \mathrm{J}$ mouse permissive to $L$. pneumophila intracellular replication. Further studies showed that flagellin sensing by wild-type NAIP5 restricts $L$. pneumophila infection (Zamboni et al., 2006; Fortier et al., 2007; Lamkanfi et al., 2007; Fortier et al., 2009) and that macrophages derived from NAIP5 knockout mice is defective in flagellin stimulation of caspase-1 activation during $L$. pneumophila infection (Lightfield et al., 2008). The biochemical assay established by Zhao et al. further revealed a universal role for NAIP5 in NLRC4 inflammasome activation in response to different bacterial flagellins (Zhao et al., 2011). In both yeast two-hybrid and co-immunoprecipitation assays, flagellin shows a robust direct interaction with NAIP5 and also the closely related NAIP6 (Zhao et al., 2011). Furthermore, the ability of flagellins from ten different bacterial pathogens to bind to NAIP5 correlates well with their differential inflammasome-stimulating activity (Zhao et al., 2011). This further strengthens the idea that flagellin is directly recognized by NAIP5 in triggering NLRC4 inflammasome activation. In fact, flagellin binding promotes a physical association between NAIP5(6) and NLRC4 (Zhao et al., 2011), resulting in formation of an oligomeric NLRC4 inflammasome complex and full reconstitution of flagellin stimulation of $\mathrm{IL}-1 \beta$ production in non-macrophage cells (Kofoed and Vance, 2011; Zhao et al., 2011). Thus, NAIP5(6) serves as the inflammasome receptor for bacterial flagellin and NLRC4 indeed functions as an adaptor that relays the NAIP5 signaling to downstream caspase-1 activation and macrophage inflammation (Fig. 1).

Flagellin can be synthesized de novo upon sensing of host-produced lysophospholipids (Subramanian and Qadri, 2006). Translocation of flagellin monomers into macrophage cytosol for inflammasome stimulation is not through the flagellar secretion system (Miao et al., 2006), and instead is mediated by bacterial type III or type IV secretion system. Therefore, flagellin stimulation of the inflammasome pathway is usually absent or significantly attenuated for bacterial mutants harboring a genetically disrupted type III or type IV secretion system. For example, deletion of sipB that encodes a key component in the type III secretion translocator pore from S. typhimurium diminishes infection-induced caspase-1 activation and macrophage inflammatory death (Hersh et al., 1999). Similarly, the L. pneumophila $\operatorname{dot} A$ mutant deficient in type IV-mediated effector translocation is also unable to stimulate inflammasome activation. Using the $\beta$-lactamase reporter secretion assay, it has been demonstrated that $S$. typhimurium flagellin can be injected into host cells through Salmonella Pathogenicity Island 1 (SPI-1) type III secretion system (Sun et al., 2007). Moreover, it is also a possibility that lysis of intracellular bacteria can lead to cytosolic presence of bacteria flagellin, which might explain activation of NLRC4 inflammasome by flagellin from $L$. monocytogenes that harbors neither type III nor type IV secretion system (Warren et al., 2008; Tsuchiya et al., 2010; Wu et al., 2010). 


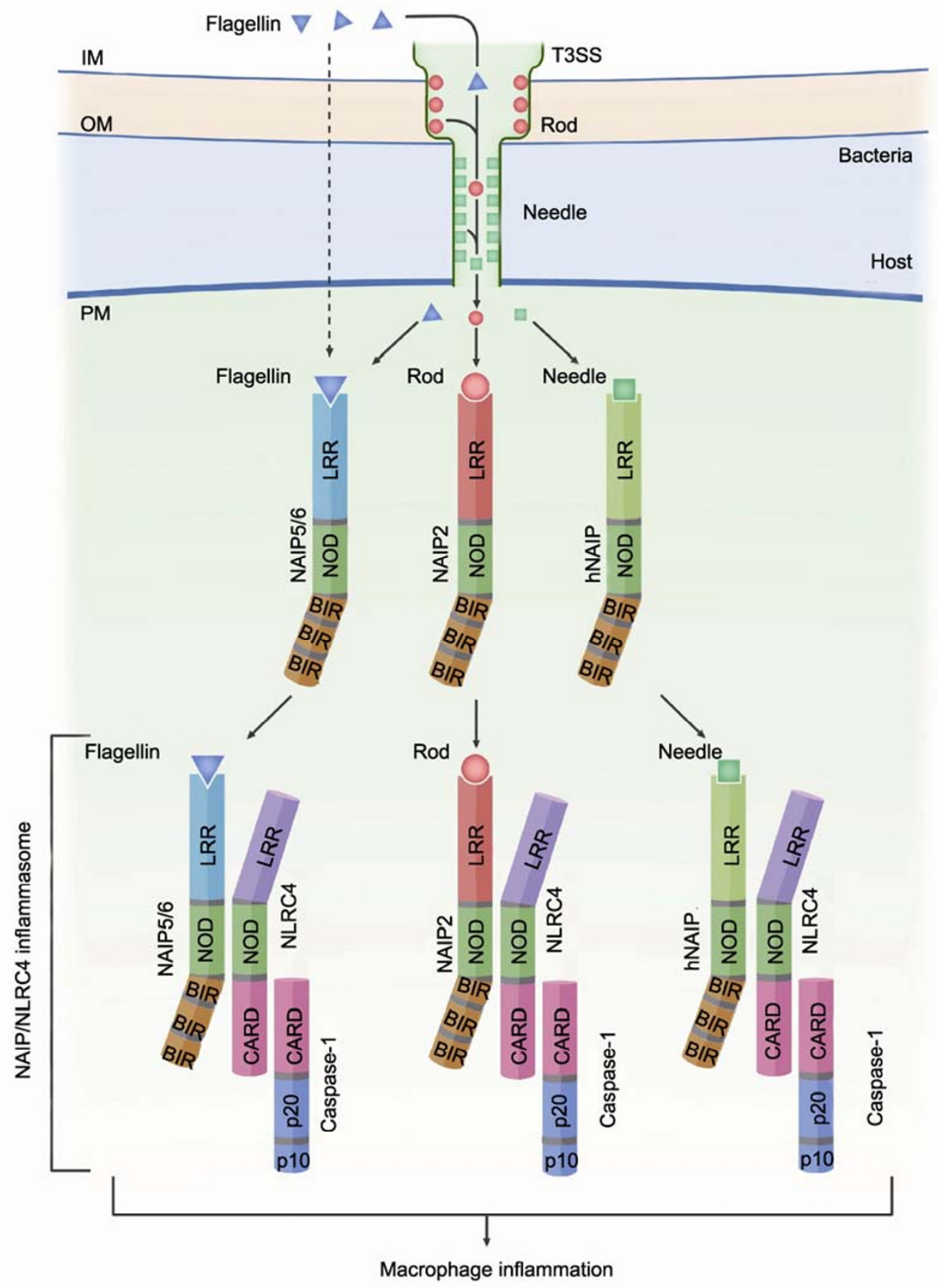

Figure 1. Model of NAIP receptors for recognizing bacterial products in stimulating NLRC4 inflammasome activation. The NAIP family of NLR proteins contains three BIR domains at the $\mathrm{N}$ termini and leucine-rich repeat (LRR) domain at the $\mathrm{C}$ termini. Murine NAIP5 (possibly also the closely related NAIP6) directly recognizes monomeric bacterial flagellin (blue triangle) that accidently gets into macrophage cytosol through the type III secretion system (T3SS) (solid line) or other T3SS-independent means (dashed line). Another murine NAIP-family member NAIP2 recognizes the rod component (red circle) of the T3SS while the sole NAIP protein in human (hNAIP) serves as the inflammasome receptor for the T3SS needle subunit (green square). Binding of NAIP receptors by corresponding bacterial ligands induces a physical interaction between NAIP and NLRC4, leading to formation of an oligomeric NLRC4 inflammasome complex. This complex serves as a platform for inducing downstream caspase-1 activation through the CARD-CARD interaction between NLRC4 and caspase-1. Caspase-1 activation initiates macrophage inflammatory responses including processing and maturation of IL-1 $\beta / \mathrm{LL}-18$ and macrophage pyroptotic cell death. PM: eukaryotic cell plasma membrane; OM: bacterial outer membrane; IM: bacterial inner membrane. 


\section{RECOGNITION OF BACTERIAL TYPE III SECR- ETION SYSTEM BY MURINE NAIP2 AND HUMAN NAIP}

Given the evolutional and functional connection between the type III secretion system and the flagellar system, it has been proposed that inflammasome sensing of a small portion of newly synthesized flagellin monomers, which are accidentally translocated into host cytosol through the type III secretion system, serves as an indirect means for the host to detect an active virulent effector secretion system (Miao and Warren, 2010). However, some bacterial pathogens such as EPEC, $C$ violaceum and $B$. thailandensis have evaded from NAIP5(6)-mediated innate immune recognition (Zhao et al., 2011), and bacterium like Yersinia enterocolitica shuts down its flagellin expression at the infection temperature of $37^{\circ} \mathrm{C}$. Therefore, evidences have been provided that the NLRC4 inflammasome can also respond to the rod subunit of the type III secretion system, as has been observed with PrgJ in Salmonella SPI-1 in mouse macrophage infection (Miao et al., 2010b). Zhao et al. further noted that the NLRC4 inflammasome in human monocytic U937 cells specifically responds to the needle subunit of the type III secretion system, such as Prgl in S. typhimurium and Cprl in C. violaceum (Zhao et al., 2011). Cytoplasmic delivery of purified recombinant type III secretion rod or needle subunit by using the $\mathrm{N}$-terminal domain of anthrax lethal factor can stimulate NLRC4-dependent caspase-1 activation and macrophage inflammation, indicating an inflammasome-stimulating activity of these secretion system components by themselves (Zhao et al., 2011). Interestingly, similarly to that observed with flagellin, the rod and needle proteins from different bacterial species also show differential inflammasome-stimulating activity. Thus, detection of both flagellin and the type III secretion apparatus components by the NLRC4 inflammasome can maximize the capacity of this arm of innate immunity to ensure an effective response to the similar type of bacterial activities.

Analogous to flagellin recognition by NAIP5(6) receptor, the rod protein is recognized by another NAIP family member, NAIP2 in mice (Kofoed and Vance, 2011; Zhao et al., 2011). Mouse genome encodes seven homologous NAIP proteins, and NAIP2 is most distantly related to NAIP5 in primary sequence. RNA interference knockdown of NAIP2 in mouse bone marrow macrophages attenuates the rod protein-triggered inflammasome activation during EPEC and $S$. typhimurium infection. NAIP2 can directly bind to the rod protein such as PrgJ of $S$. typhimurium and BsaK of $B$. thailandensis (Zhao et al., 2011), and this binding also stimulates a complex formation between NAIP2 and NLRC4 (Fig. 1), rendering functional reconstitution of an oligomeric NLRC4 inflammasome complex in nonmacrophage cells (Kofoed and Vance., 2011; Zhao et al., 2011). Human only harbors one
NAIP family member and human monocytic U937 cells are defective in sensing bacterial flagellin and the type III secretion rod subunit (Zhao et al., 2011). Genetic studies of $C$. violaceum infection of U937 cells revealed that the type III secretion needle subunit $\mathrm{Cprl}$ is responsible for infection-triggered NLRC4 inflammasome activation. Biochemically, both Cprl and Prgl of S. typhimurium are proficient in direct interaction with human NAIP, suggesting that human NAIP functions in a similar way as NAIP5(6)/2 in mice and serves as the receptor for the type III secretion needle subunit (Fig. 1) (Zhao et al., 2011). Furthermore, human NAIP, but not murine NAIP5(6) and NAIP2, supports reconstitution of an active NLRC4 inflammasome that can specifically respond to intracellular delivery of recombinant Cprl or Prgl. Other homologous needle subunits from EHEC, B. thailandensis, $P$. aeruginosa, $S$. flexneri and $S$. typhimurium can also stimulate NLRC4 inflammasome activation in human cells (Zhao et al., 2011). This suggests that direct recognition of the type III secretion needle subunits by human NAIP represents another general innate immune response to bacterial infection.

Previous studies on the mechanism of type III secretion suggest that both the rod and needle subunits are secreted prior to initiation of effector secretion. The rod protein could also be accidently exported out of the bacteria. This provides an explanation for how these secretion apparatus components can be exposed to recognition by the NAIP receptors in macrophage cytosol. This is certainly the case for some bacterial pathogens. For example, mutation of the essential ATPase component in S. typhimurium SPI-1 blocks $S$. typhimurium-induced inflammasome activation in human U937 cells that results from activity of the needle subunit Prgl. However, the situation appears to be different for $C$. violaceum infection as the needle protein Cprl-triggered caspase-1 activation in U937 cells still occurs in the absence of the two type III secretion ATPase subunits (Zhao et al., 2011). This indicates that entry of the needle subunit into macrophage cytosol does not absolutely require a functional type III secretion system. Consistently, ectopic expression of S. typhimurium SPI-1 rod protein PrgJ in L. monocytogenes can potently stimulate NAIP2-dependent NLRC4 inflammasome activation (Kofoed and Vance., 2011). Therefore, the rod and needle proteins can get into macrophage cytosol in both type III secretion-dependent and -independent manners (for instance, bacterial lysis), and the relative contribution of each channel depends on the particular pathogen and likely also the infection condition.

\section{CONCLUSION AND PERSPECTIVE}

Thus, in addition to sensing cytoplasmic presence of bacterial flagellin by the NAIP5 receptor, the innate immune system in mammals employ other NAIP proteins such as NAIP2 in mice and NAIP in human to directly recognize components of 
bacterial type III secretion system. Engagement of NAIP receptors by corresponding bacterial ligands promotes their physical interaction with NLRC4 and formation of an oligomeric NLRC4 inflammasome complex, resulting in activation of caspase-1 and macrophage innate immunity (Fig. 1). Similarly to that of flagellin, the inflammasome-stimulating activities of type III secretion rod and needle proteins also reside in their C-terminal helical hairpin regions that are enriched in hydrophobic residues. Therefore, other homologous NAIP family members in mice might recognize bacterial products of similar biochemical features. The arrival of seven NAIPs in the mouse system, probably resulting from gene duplication during the evolution, likely reflects the need of a broad-spectrum innate immune surveillance for the mouse to cope with diverse bacterial infections it encounters. Moreover, it is also interesting to note here that the NLR-like protein Apaf-1 recognizes mitochondrion-derived cytochrome $c$ to form the oligomeric apoptosome complex for caspase- 9 activation, particularly given the evolutional connection between mitochondria and bacteria.

Two important questions remain regarding the mechanism and other functional aspects of the NAIP-NLRC4 inflammasome axis in anti-bacterial innate immunity. Firstly, what is the biochemical basis for specific recognition of flagellin and the type III secretion rod and needle proteins by different NAIP receptors? Answer to this question requires future structural studies of NAIP receptors in complex with the particular bacterial ligand. Secondly, are there additional functions of the NLRC4 inflammasome in innate immune detection of other bacterial infection? For example, genetic deletion of zmp1 from Mycobacterium tuberculosis, which encodes a putative metalloprotease, results in elevated inflammasome activation that is mediated by NLRC4 and ASC (Master et al., 2008). This suggests that the NLRC4 inflammasome likely detects an unknown signal from $M$. tuberculosis. It is worthwhile to further probe whether this activation also involves a NAIP family receptor and what is the biochemical mechanism underlying Zmp1 inhibition of NLRC4 inflammasome activation.

\section{ACKNOWLEDGEMENTS}

Work in the laboratory of FS was supported by the National Basic Research Program of China (973 Program) (Grant Nos. 2010CB835400 and 2012CB518700) and Howard Hughes Medical Institute, USA.

\section{ABBREVIATIONS}

CARD, caspase recruitment domain; EHEC, enterohemorrhagic $E$. coli; EPEC, enteropathogenic E. coli; LRR, leucine-rich repeat; NLRs, NOD-like receptors; NOD, oligomerization domain; PAMPs, pathogen-associated molecular patterns; PRRs, pattern recognition receptors; TLRs, Toll-like receptors.

\section{REFERENCES}

Amer, A., Franchi, L., Kanneganti, T.D., Body-Malapel, M., Ozören, N., Brady, G., Meshinchi, S., Jagirdar, R., Gewirtz, A., Akira, S., et al. (2006). Regulation of Legionella phagosome maturation and infection through flagellin and host Ipaf. J Biol Chem 281, 35217-35223.

Anand, P.K., Malireddi, R.K., and Kanneganti, T.D. (2011). Role of the nirp3 inflammasome in microbial infection. Front Microbiol 2,.

Boyden, E.D., and Dietrich, W.F. (2006). Nalp1b controls mouse macrophage susceptibility to anthrax lethal toxin. Nat Genet 38 , 240-244.

Brodsky, I.E., and Medzhitov, R. (2011). Pyroptosis: macrophage suicide exposes hidden invaders. Curr Biol 21, R72-R75.

Broz, P., Newton, K., Lamkanfi, M., Mariathasan, S., Dixit, V.M., and Monack, D.M. (2010a). Redundant roles for inflammasome receptors NLRP3 and NLRC4 in host defense against Salmonella. J Exp Med 207, 1745-1755.

Broz, P., von Moltke, J., Jones, J.W., Vance, R.E., and Monack, D.M. (2010b). Differential requirement for Caspase-1 autoproteolysis in pathogen-induced cell death and cytokine processing. Cell Host Microbe 8, 471-483.

Case, C.L., Shin, S., and Roy, C.R. (2009). Asc and Ipaf Inflammasomes direct distinct pathways for caspase-1 activation in response to Legionella pneumophila. Infect Immun 77, 1981-1991.

Chen, G., Shaw, M.H., Kim, Y.G., and Nuñez, G. (2009). NOD-like receptors: role in innate immunity and inflammatory disease. Annu Rev Pathol 4, 365-398.

Chen, G.Y., Liu, M., Wang, F., Bertin, J., and Núñez, G. (2011). A functional role for Nlrp6 in intestinal inflammation and tumorigenesis. J Immunol 186, 7187-7194.

Chen, L.M., Kaniga, K., and Galán, J.E. (1996). Salmonella spp. are cytotoxic for cultured macrophages. Mol Microbiol 21, 1101-1115.

Cornelis, G.R. (2010). The type III secretion injectisome, a complex nanomachine for intracellular 'toxin' delivery. Biol Chem 391, 745-751.

Cui, J., and Shao, F. (2011). Biochemistry and cell signaling taught by bacterial effectors. Trends Biochem Sci 36, 532-540.

Davis, B.K., Wen, H., and Ting, J.P. (2011). The inflammasome NLRs in immunity, inflammation, and associated diseases. Annu Rev Immunol 29, 707-735.

Deane, J.E., Roversi, P., Cordes, F.S., Johnson, S., Kenjale, R., Daniell, S., Booy, F., Picking, W.D., Picking, W.L., Blocker, A.J., et al. (2006). Molecular model of a type III secretion system needle: Implications for host-cell sensing. Proc Natl Acad Sci U S A 103, 12529-12533.

Derré, I., and Isberg, R.R. (2004). Macrophages from mice with the restrictive Lgn1 allele exhibit multifactorial resistance to Legionella pneumophila. Infect Immun 72, 6221-6229.

Diez, E., Lee, S.H., Gauthier, S., Yaraghi, Z., Tremblay, M., Vidal, S., and Gros, P. (2003). Birc1e is the gene within the Lgn1 locus associated with resistance to Legionella pneumophila. Nat Genet 33, 55-60.

Duncan, J.A., Bergstralh, D.T., Wang, Y., Willingham, S.B., Ye, Z., 
Zimmermann, A.G., and Ting, J.P. (2007). Cryopyrin/NALP3 binds ATP/dATP, is an ATPase, and requires ATP binding to mediate inflammatory signaling. Proc Natl Acad Sci U S A 104, 8041-8046.

Elinav, E., Strowig, T., Kau, A.L., Henao-Mejia, J., Thaiss, C.A., Booth, C.J., Peaper, D.R., Bertin, J., Eisenbarth, S.C., Gordon, J.I., et al. (2011). NLRP6 inflammasome regulates colonic microbial ecology and risk for colitis. Cell 145, 745-757.

Erhardt, M., Namba, K., and Hughes, K.T. (2010). Bacterial nanomachines: the flagellum and type III injectisome. Cold Spring Harb Perspect Biol 2, a000299.

Faustin, B., Lartigue, L., Bruey, J.M., Luciano, F., Sergienko, E., Bailly-Maitre, B., Volkmann, N., Hanein, D., Rouiller, I., and Reed, J.C. (2007). Reconstituted NALP1 inflammasome reveals two-step mechanism of caspase-1 activation. Mol Cell 25, 713-724.

Fink, S.L., and Cookson, B.T. (2006). Caspase-1-dependent pore formation during pyroptosis leads to osmotic lysis of infected host macrophages. Cell Microbiol 8, 1812-1825.

Fortier, A., de Chastellier, C., Balor, S., and Gros, P. (2007). Birc1e/Naip5 rapidly antagonizes modulation of phagosome maturation by Legionella pneumophila. Cell Microbiol 9, 910-923.

Fortier, A., Doiron, K., Saleh, M., Grinstein, S., and Gros, P. (2009). Restriction of Legionella pneumophila replication in macrophages requires concerted action of the transcriptional regulators IIf1 and Irf8 and nod-like receptors Naip5 and Nlrc4. Infect Immun 77, 4794-4805.

Franchi, L., Amer, A., Body-Malapel, M., Kanneganti, T.D., Ozören, N., Jagirdar, R., Inohara, N., Vandenabeele, P., Bertin, J., Coyle, A., et al. (2006). Cytosolic flagellin requires Ipaf for activation of caspase-1 and interleukin 1beta in salmonella-infected macrophages. Nat Immunol 7, 576-582.

Franchi, L., Stoolman, J., Kanneganti, T.D., Verma, A., Ramphal, R., and Núñez, G. (2007). Critical role for Ipaf in Pseudomonas aeruginosa-induced caspase-1 activation. Eur J Immunol 37, 3030-3039.

Galán, J.E., and Wolf-Watz, H. (2006). Protein delivery into eukaryotic cells by type III secretion machines. Nature 444, 567-573.

Galle, M., Schotte, P., Haegman, M., Wullaert, A., Yang, H.J., Jin, S., and Beyaert, R. (2008). The Pseudomonas aeruginosa Type III secretion system plays a dual role in the regulation of caspase- 1 mediated IL-1beta maturation. J Cell Mol Med 12, 1767-1776.

Ge, J. and Shao, F. (2011). Manipulation of host vesicular trafficking and innate immune defense by Legionella Dot/lcm effectors. Cell Microbiol 13, 1870-1880.

Hersh, D., Monack, D.M., Smith, M.R., Ghori, N., Falkow, S., and Zychlinsky, A. (1999). The Salmonella invasin SipB induces macrophage apoptosis by binding to caspase-1. Proc Natl Acad Sci U S A 96, 2396-2401.

Hornung, V., and Latz, E. (2010). Intracellular DNA recognition. Nat Rev Immunol 10, 123-130.

Isberg, R.R., O'Connor, T.J., and Heidtman, M. (2009). The Legionella pneumophila replication vacuole: making a cosy niche inside host cells. Nat Rev Microbiol 7, 13-24.

Kofoed, E.M., and Vance, R.E. (2011). Innate immune recognition of bacterial ligands by NAIPs determines inflammasome specificity. Nature 477, 592-595.

Lamkanfi, M., Amer, A., Kanneganti, T.D., Muñoz-Planillo, R., Chen, G., Vandenabeele, P., Fortier, A., Gros, P., and Núñez, G. (2007). The Nod-like receptor family member Naip5/Birc1e restricts Legionella pneumophila growth independently of caspase-1 activation. J Immunol 178, 8022-8027.

Lightfield, K.L., Persson, J., Brubaker, S.W., Witte, C.E., von Moltke, J., Dunipace, E.A., Henry, T., Sun, Y.H., Cado, D., Dietrich, W.F., et al. (2008). Critical function for Naip5 in inflammasome activation by a conserved carboxy-terminal domain of flagellin. Nat Immunol 9, 1171-1178.

Mariathasan, S., Newton, K., Monack, D.M., Vucic, D., French, D.M., Lee, W.P., Roose-Girma, M., Erickson, S., and Dixit, V.M. (2004). Differential activation of the inflammasome by caspase- 1 adaptors ASC and Ipaf. Nature 430, 213-218.

Marlovits, T.C., Kubori, T., Lara-Tejero, M., Thomas, D., Unger, V.M., and Galán, J.E. (2006). Assembly of the inner rod determines needle length in the type III secretion injectisome. Nature 441, $637-640$.

Master, S.S., Rampini, S.K., Davis, A.S., Keller, C., Ehlers, S., Springer, B., Timmins, G.S., Sander, P., and Deretic, V. (2008). Mycobacterium tuberculosis prevents inflammasome activation. Cell Host Microbe 3, 224-232.

Miao, E.A., Alpuche-Aranda, C.M., Dors, M., Clark, A.E., Bader, M.W., Miller, S.I., and Aderem, A. (2006). Cytoplasmic flagellin activates caspase-1 and secretion of interleukin 1 beta via Ipaf. Nat Immunol 7, 569-575.

Miao, E.A., Ernst, R.K., Dors, M., Mao, D.P., and Aderem, A. (2008). Pseudomonas aeruginosa activates caspase 1 through Ipaf. Proc Natl Acad Sci U S A 105, 2562-2567.

Miao, E.A., Leaf, I.A., Treuting, P.M., Mao, D.P., Dors, M., Sarkar, A., Warren, S.E., Wewers, M.D., and Aderem, A. (2010a). Caspase-1-induced pyroptosis is an innate immune effector mechanism against intracellular bacteria. Nat Immunol 11, 1136-1142.

Miao, E.A., Mao, D.P., Yudkovsky, N., Bonneau, R., Lorang, C.G., Warren, S.E., Leaf, I.A., and Aderem, A. (2010b). Innate immune detection of the type III secretion apparatus through the NLRC4 inflammasome. Proc Natl Acad Sci U S A 107, 3076-3080.

Miao, E.A., and Warren, S.E. (2010). Innate immune detection of bacterial virulence factors via the NLRC4 inflammasome. J Clin Immunol 30, 502-506.

Molofsky, A.B., Byrne, B.G., Whitfield, N.N., Madigan, C.A., Fuse, E.T., Tateda, K., and Swanson, M.S. (2006). Cytosolic recognition of flagellin by mouse macrophages restricts Legionella pneumophila infection. J Exp Med 203, 1093-1104.

Ren, T., Zamboni, D.S., Roy, C.R., Dietrich, W.F., and Vance, R.E. (2006). Flagellin-deficient Legionella mutants evade caspase-1and Naip5-mediated macrophage immunity. PLoS Pathog 2, e18.

Schroder, K., and Tschopp, J. (2010). The inflammasomes. Cell 140, 821-832.

Subramanian, N., and Qadri, A. (2006). Lysophospholipid sensing 
triggers secretion of flagellin from pathogenic salmonella. Nat Immunol 7, 583-589.

Sun, Y.H., Rolán, H.G., and Tsolis, R.M. (2007). Injection of flagellin into the host cell cytosol by Salmonella enterica serotype Typhimurium. J Biol Chem 282, 33897-33901.

Sutterwala, F.S., Mijares, L.A., Li, L., Ogura, Y., Kazmierczak, B.I., and Flavell, R.A. (2007). Immune recognition of Pseudomonas aeruginosa mediated by the IPAF/NLRC4 inflammasome. J Exp Med 204, 3235-3245.

Takeuchi, O., and Akira, S. (2010). Pattern recognition receptors and inflammation. Cell 140, 805-820.

Ting, J.P., Kastner, D.L., and Hoffman, H.M. (2006). CATERPILLERs, pyrin and hereditary immunological disorders. Nat Rev Immunol 6, 183-195.

Tsuchiya, K., Hara, H., Kawamura, I., Nomura, T., Yamamoto, T., Daim, S., Dewamitta, S.R., Shen, Y., Fang, R., and Mitsuyama, M. (2010). Involvement of absent in melanoma 2 in inflammasome activation in macrophages infected with Listeria monocytogenes. J Immunol 185, 1186-1195.
Warren, S.E., Mao, D.P., Rodriguez, A.E., Miao, E.A., and Aderem, A. (2008). Multiple Nod-like receptors activate caspase 1 during Listeria monocytogenes infection. J Immunol 180, 7558-7564.

Wright, E.K., Goodart, S.A., Growney, J.D., Hadinoto, V., Endrizzi, M.G., Long, E.M., Sadigh, K., Abney, A.L., Bernstein-Hanley, I., and Dietrich, W.F. (2003). Naip5 affects host susceptibility to the intracellular pathogen Legionella pneumophila. Curr Biol 13, 27-36.

Wu, J., Fernandes-Alnemri, T., and Alnemri, E.S. (2010). Involvement of the AIM2, NLRC4, and NLRP3 inflammasomes in caspase-1 activation by Listeria monocytogenes. J Clin Immunol 30, 693-702.

Zamboni, D.S., Kobayashi, K.S., Kohlsdorf, T., Ogura, Y., Long, E.M., Vance, R.E., Kuida, K., Mariathasan, S., Dixit, V.M., Flavell, R.A., et al. (2006). The Birc1e cytosolic pattern-recognition receptor contributes to the detection and control of Legionella pneumophila infection. Nat Immunol 7, 318-325.

Zhao, Y., Yang, J., Shi, J., Gong, Y.N., Lu, Q., Xu, H., Liu, L., and Shao, F. (2011). The NLRC4 inflammasome receptors for bacterial flagellin and type III secretion apparatus. Nature 477, 596-600. 